\title{
Optical pumping of a single hole spin in a quantum dot
}

\author{
Brian D. Gerardot ${ }^{1}$, Daniel Brunner ${ }^{1}$, Paul A. Dalgarno ${ }^{1}$, Patrik Öhberg ${ }^{1}$, Stefan Seidl ${ }^{2}$, Martin Kroner $^{2}$, \\ Khaled Karrai ${ }^{2}$, Nick G. Stoltz ${ }^{3}$, Pierre M. Petroff ${ }^{3} \&$ Richard J. Warburton $^{1}$
}

The spin of an electron is a natural two-level system for realizing a quantum bit in the solid state ${ }^{1-16}$. For an electron trapped in a semiconductor quantum dot, strong quantum confinement highly suppresses the detrimental effect of phonon-related spin relaxation $^{1-7}$. However, this advantage is offset by the hyperfine interaction between the electron spin and the $10^{4}$ to $10^{6}$ spins of the host nuclei in the quantum dot. Random fluctuations in the nuclear spin ensemble lead to fast spin decoherence in about ten nanoseconds ${ }^{8-14}$. Spin-echo techniques have been used to mitigate the hyperfine interaction ${ }^{14,15}$, but completely cancelling the effect is more attractive. In principle, polarizing all the nuclear spins can achieve this ${ }^{16,17}$ but is very difficult to realize in practice ${ }^{12,18,19}$. Exploring materials with zero-spin nuclei is another option, and carbon nanotubes ${ }^{20}$, graphene quantum dots ${ }^{21}$ and silicon have been proposed. An alternative is to use a semiconductor hole. Unlike an electron, a valence hole in a quantum dot has an atomic $p$ orbital which conveniently goes to zero at the location of all the nuclei, massively suppressing the interaction with the nuclear spins. Furthermore, in a quantum dot with strong strain and strong quantization, the heavy hole with spin-3/2 behaves as a spin-1/2 system and spin decoherence mechanisms are weak ${ }^{22,23}$. We demonstrate here high fidelity (about 99 per cent) initialization of a single hole spin confined to a self-assembled quantum dot by optical pumping. Our scheme works even at zero magnetic field, demonstrating a negligible hole spin hyperfine interaction. We determine a hole spin relaxation time at low field of about one millisecond. These results suggest a route to the realization of solid-state quantum networks ${ }^{24}$ that can intra-convert the spin state with the polarization of a photon.

Our scheme to initialize a single hole spin is presented in Fig. 1. The quantum dot contains a single hole. The strong in-built strain in an InAs quantum dot shifts the valence light hole states with spin $J=3 / 2$, $J_{z}= \pm 1 / 2$ away from the fundamental gap such that the uppermost valence states have heavy hole character with spin $J=3 / 2, J_{z}= \pm 3 / 2$. The corresponding hole spin states are represented as $|\Uparrow\rangle$ and $|\Downarrow\rangle . \mathrm{A} \sigma^{+}$ -polarized laser drives the $|\Downarrow\rangle$ hole to an exciton state with spin $S_{z}=-1 / 2,|\Uparrow \downarrow, \downarrow\rangle$, containing a spin-up, spin-down hole pair and a spin-down electron. Unlike the hole spin, the electron spin interacts with the nuclear spins through the contact hyperfine interaction. The electron spin experiences a small magnetic field, $\sim 20 \mathrm{mT}$ (refs 8-12), as a result of the incomplete cancellation of the random nuclear spins in the quantum dot. The component of the magnetic field in the plane, $B_{\text {nuclei }}^{x y}$, causes the electron spin in the excited state to precess with a period of $\sim 1 \mathrm{~ns}$. The coherence of the precession is destroyed by spontaneous emission with a characteristic time of $\sim 1 \mathrm{~ns}$ and results in the shelving of hole spin in the $|\Uparrow\rangle$ state, which does not couple to the laser field, according to the spin selection rule. This process initializes the hole spin with high fidelity provided that the hole spin relaxation time is sufficiently large. Rather than a hindrance, the hyperfine interaction between the electron spin and nuclear spins is beneficial in this scheme because it enables fast hole spin initialization. The scheme works even when the spin states are degenerate. This is advantageous because hole spin flip processes involving a single phonon are turned off in the limit of zero magnetic field ${ }^{22}$. The overall scheme depends on stringent requirements: a very clean optical selection rule, slow hole spin relaxation, and negligible interaction between the hole spin and the nuclear spins. We report here successful implementation of this concept with very high fidelity even at zero applied magnetic field where the hole spin is shielded from the laser only by its spin and not by an energy detuning. We confirm our interpretation by applying a magnetic field along the quantization axis $\mathbf{z}$ which slows down the precession, gradually turning off the hole spin initialization process.

Our sample, grown by molecular beam epitaxy, consists of InGaAs quantum dots embedded in the intrinsic region of a metal-insulatorsemiconductor field electron transistor (Fig. 2a), allowing deterministic charging. The quantum dots are separated by $25 \mathrm{~nm}$ from a hole Fermi sea. With an applied bias between a semi-transparent Schottky gate and the Fermi sea, the quantum dot energy can be controlled relative to the Fermi energy thus determining the charge state. Initial characterization is carried out using photoluminescence spectroscopy (Fig. 2b). Each discrete jump in the emission spectrum signals a charging event and we can identify the charge of each state by the characteristic fine structures ${ }^{25,26}$. The overlap in gate voltage for different charge states is a consequence of the relatively large hole tunnelling time, which we estimate to be $\sim 10 \mathrm{ns,} \mathrm{compared} \mathrm{to} \mathrm{the}$ radiative emission lifetime of $0.8 \mathrm{~ns}$. This is crucial: it ensures that the cotunnelling process ${ }^{6,7,27}$ of hole spin relaxation through spin-swap with the Fermi sea is sufficiently weak to implement our scheme.

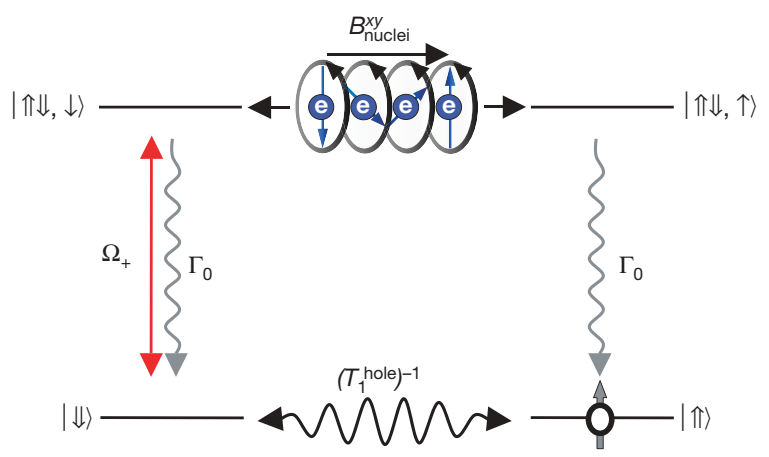

Figure 1 | Scheme to initialize a single hole spin at zero magnetic field. The two hole spin states, $|\Downarrow\rangle$ and $|\Uparrow\rangle$, and the two exciton spin states, $|\Uparrow \downarrow, \downarrow\rangle$ and $|\uparrow \Downarrow, \uparrow\rangle$ are depicted with coherent couplings (solid lines) and incoherent couplings (wavy lines). Open (or solid) arrows depict hole (or electron) spins. The dipole transition from $|\downarrow\rangle$ to $|\Uparrow \downarrow, \downarrow\rangle$ is driven on-resonance with a $\sigma^{+}$-polarized laser $\left(\Omega_{+}\right)$; electron spin (blue 'e' symbols) precession in the effective magnetic field generated by the nuclear spins coherently couples the $|\Uparrow \downarrow, \downarrow\rangle$ and $|\Uparrow \Downarrow, \uparrow\rangle$ states. Through spontaneous recombination $\left(\Gamma_{0}\right)$, the population is shelved in the $|\Uparrow\rangle$ state. 
a

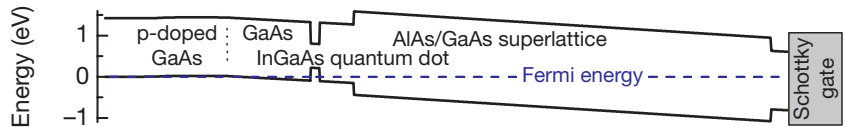

b

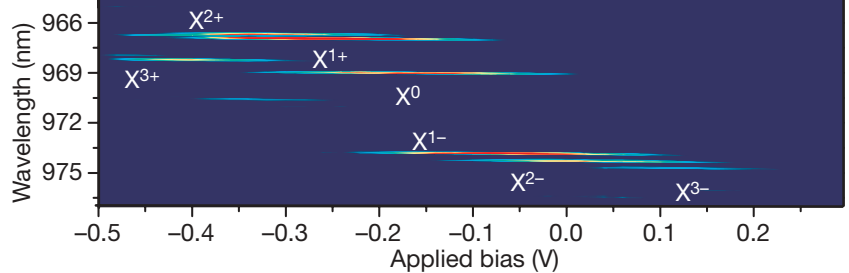

C
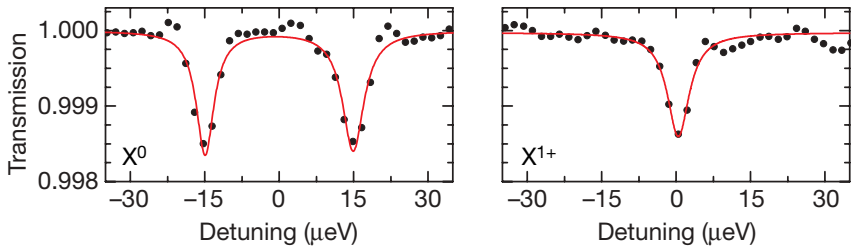

Figure 2 | Experimental methods to probe a single hole spin. a, The band diagram for our device. Holes tunnel through a $25 \mathrm{~nm}$ barrier into the selfassembled InGaAs quantum dots from the p-doped (carbon doping) back contact. An applied bias between the semi-transparent top gate controls the quantum dot charge state. $\mathbf{b}$, Photoluminescence from a single quantum dot shows pronounced Coulomb blockade as the applied voltage is varied. Each spectral jump corresponds to a charging event. c, Resonant laser spectroscopy of the empty $\left(\mathrm{X}^{0}\right)$ and singly positively charged $\left(\mathrm{X}^{1+}\right)$ states for the same quantum dot. The detuning is achieved with the applied bias via the Stark effect. The laser has linear polarization oriented at $45^{\circ}$ to the crystallographic axis. This polarization equally pumps the two spin transitions for the $\mathrm{X}^{1+}$ transition and also the two transitions in the fine structure of the $\mathrm{X}^{0}$ transition. $1 \mathrm{nW}$ of power is used in each experiment and the sample temperature is $4.2 \mathrm{~K}$. The solid lines are lorentzian fits to the data.

We drive the optical transitions with a highly coherent continuous-wave laser, detecting resonant scattering of the laser light from the quantum dot by measuring the transmission coefficient with very high signal-to-noise ratio $^{26,28}$. Figure $2 \mathrm{c}$ displays transmission curves as the quantum dot transition energy is tuned through the laser energy via the Stark effect when the quantum dot is empty $\left(\mathrm{X}^{0}\right.$ transition) and charged with a single hole $\left(\mathrm{X}^{1+}\right.$ transition), in both cases using linear polarization. We observe lorentzian lineshapes with linewidths of $\sim 5 \mu \mathrm{eV}$. This is larger than both the radiationbroadened linewidth $(0.8 \mu \mathrm{eV})$ and the linewidth observed in samples with n-doped back contacts $(1-2 \mu \mathrm{eV})^{6,7,26,28}$, but small enough to record high-quality laser spectroscopy data. The increase in linewidth beyond the radiative limit is caused by a spectral fluctuation (see Supplementary Information).

Our main result is revealed in the transmission spectra taken at zero externally applied magnetic field, $B_{\text {ext }}^{z}=0$, when the two hole spin states are degenerate. We find that the contrast, defined as the size of the transmission dip at zero detuning, is immeasurably small when the exciting laser has circular polarization, either $\sigma^{+}$(Fig. 3a) or $\sigma^{-}$(Fig. 3b). This suggests that the hole is prohibited by its spin from scattering the laser light, that is, that optical spin pumping is taking place. We prove this by pumping with two lasers with identical wavelength and with the same total power, one with $\sigma^{+}$and one with $\sigma^{-}$polarization. In this case, a clear transmission dip appears (Fig. 3c). This arises because spin pumping with $\sigma^{+}$polarization is frustrated by the $\sigma^{-}$excitation, and vice versa, a repumping phenomenon ${ }^{6}$.

Hole spin pumping at small magnetic fields is also highly effective. In the Faraday configuration, the degeneracy of the two optical transitions is lifted by the sum of the electron and hole Zeeman energies. When the $\sigma^{+}$-polarized laser is tuned to the $|\Downarrow\rangle \leftrightarrow|\Uparrow \downarrow, \downarrow\rangle$ resonance, the contrast is very small, signifying spin pumping into the $|\Uparrow\rangle$ state. Contrast reappears in a two-colour experiment when the $\sigma^{-}$ -polarized laser is tuned to the $|\Uparrow\rangle \leftrightarrow|\uparrow \downarrow, \uparrow\rangle$ resonance. Repumping is explored more closely in Fig. 3d. Here the maximum contrast is measured as the laser with $\sigma^{-}$polarization is detuned relative to the $|\Uparrow\rangle \leftrightarrow|\uparrow \Downarrow, \uparrow\rangle$ transition. As expected, maximum contrast is observed when the $\sigma^{-}$laser is on resonance. However, an asymmetry is observed with the repumping signal persisting more at larger than smaller detunings. The spin pumping results demonstrate that the hole spin relaxation time must be much, much larger than the spin

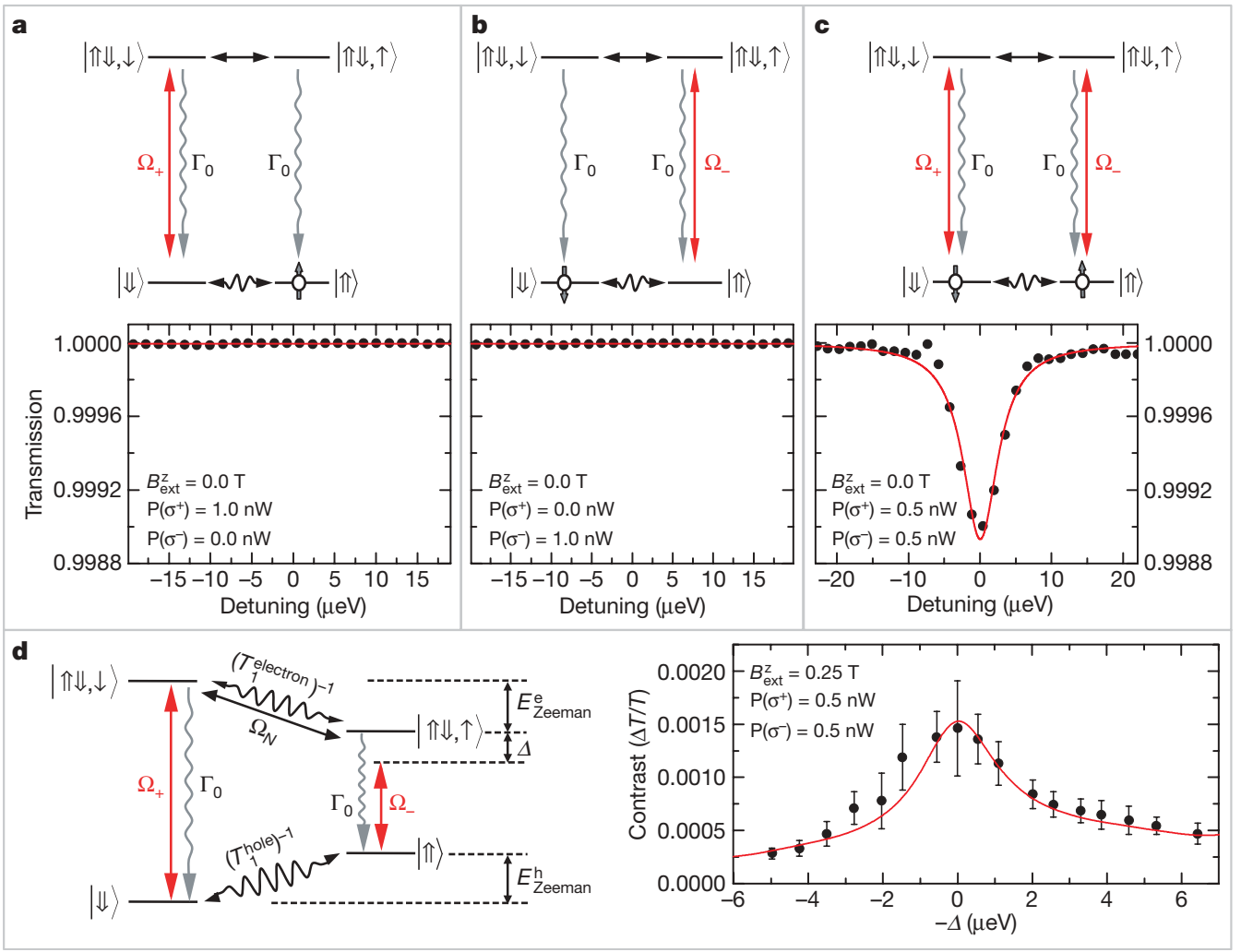

Figure 3 | Experimental demonstration of hole spin pumping. a, A laser with $\sigma^{+}$ polarization and power $\mathbf{p}\left(\sigma^{+}\right)$ drives the $|\Downarrow\rangle \leftrightarrow|\Uparrow \downarrow, \downarrow\rangle$ transition. Any transmission dip lies below the experimental signal-to-noise level. The hole population is shelved in state $|\uparrow\rangle . \mathbf{b}$, A laser with $\sigma$ polarization and power $\mathrm{p}\left(\sigma^{-}\right)$ drives the $|\Uparrow\rangle \leftrightarrow|\Uparrow \Downarrow, \uparrow\rangle$ transition. Again, any transmission dip lies below the noise floor. The hole population is shelved in state $|\Downarrow\rangle$. c, Simultaneous excitation with both $\sigma^{+}$and $\sigma^{-}$at the same frequency. A large transmission dip is observed, signifying a frustration of the spin pumping. In a-c, the solid line is a lorentzian fit to the data. d, Twocolour excitation of the Zeeman split transitions ( $E_{\text {Zeeman }}^{\mathrm{h}, \mathrm{e}}$, where e or $\mathrm{h}$ is for the electron or hole) at $B_{\text {ext }}^{z}=0.25 \mathrm{~T}$. The $\sigma^{+}$laser is onresonance with the $|\Downarrow\rangle \leftrightarrow|\Uparrow \downarrow, \downarrow\rangle$ transition while the $\sigma^{-}$laser is swept through the $|\Uparrow\rangle \leftrightarrow|\Uparrow \downarrow, \uparrow\rangle$ transition. The solid line is the result of the four-level theoretical model using $B_{\text {nuclei }}^{x y}=25 \mathrm{mT}$ and $T_{1}^{\text {hole }}=250 \mu \mathrm{s}$ for this quantum dot. The error bars correspond to s.d. for $n=10$. 
precession time. For the electron spin at fields $B_{\mathrm{ext}}^{z} \leq 0.3 \mathrm{~T}$, spin pumping is prohibited due to the hyperfine interaction ${ }^{6,7}$. This is not the case for holes: the high fidelity hole spin pumping even at $B_{\mathrm{ext}}^{z}=0$ demonstrates a negligible hole spin hyperfine interaction.

To investigate the mechanisms involved in the spin pumping process, we measure the contrast as a function of $B_{\text {ext }}^{z}$ for one-colour excitation with both $\sigma^{+}$and $\sigma^{-}$polarizations (Fig. 4a). The first feature observed is that the contrast increases as $B_{\text {ext }}^{z}$ increases. This is consistent with our proposed scheme. As $B_{\text {ext }}^{z}$ increases, the total magnetic field experienced by the electron spin is tilted further from the $(x, y)$-plane, reducing the electron spin precession rate and hence the spin pumping rate. When $B_{\mathrm{ext}}^{z}>>B_{\text {nuclei }}^{x y}$, the total magnetic field lies close to the $\mathbf{z}$-direction and spin pumping becomes very slow, eventually becoming comparable to the hole spin relaxation rate. In this regime at $\sim 3 \mathrm{~T}$, the transmission signal is fully established. A second feature of the magnetic field dependence is the increasing contrast inequality for excitation with $\sigma^{+}$relative to $\sigma^{-}$polarization. This dependence arises as the system moves gradually towards thermal equilibrium. The thermal population of $|\Downarrow\rangle$ (or $|\uparrow\rangle)$ increases (or decreases) with increasing Zeeman splitting and the experimental contrast for $\sigma^{+}$(or $\sigma^{-}$) polarization tends to increase (or decrease) at high magnetic fields.

A theoretical analysis provides a quantum mechanical description of the optical pumping scheme and a means of extracting a fidelity for the hole spin initialization and the hole spin relaxation time, $T_{1}^{\text {hole }}$. The model takes a four-level basis with the coherent couplings shown
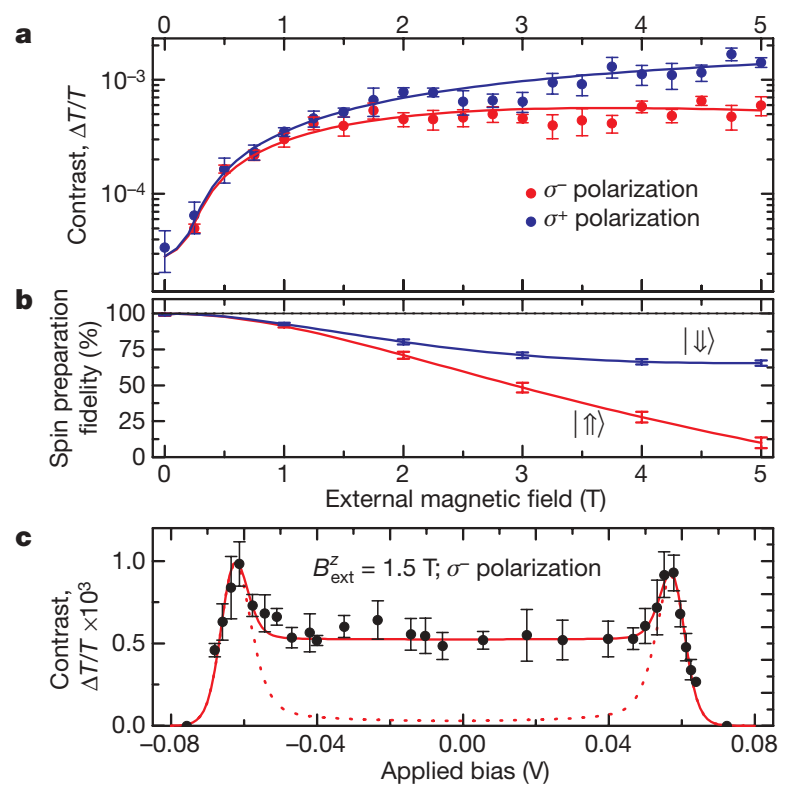

Figure 4 | Dependence of optical spin pumping on magnetic field and applied bias. a, Measured contrast for one-colour excitation, either $\sigma^{+}$or $\sigma^{-}$polarization, as a function of external magnetic field applied in the z-direction. The solid lines represent a fit to the results of the four-level theoretical calculation using $B_{\text {nuclei }}^{x y}=21 \mathrm{mT}, E_{\text {Zeeman }}^{e}=35 \mu \mathrm{eV} \mathrm{T}^{-1}$ $\left(g_{e}=-0.66\right), E_{\text {Zeeman }}^{h}=70 \mu \mathrm{eV} \mathrm{T}^{-1}, \hbar \Omega_{N}=0.73 \mu \mathrm{eV}, T=4.2 \mathrm{~K}$, $T_{1}^{\text {electron }}=10 \mathrm{~ms}$, $T_{1}^{\text {hole }}=1 \mathrm{~ms}$, and $\alpha_{0}=0.025$. Only $T_{1}^{\text {hole }}$ was used as a fitting parameter. For $\sigma^{+}$polarization, $\hbar \Omega_{+}=0.38 \mu \mathrm{eV}, \hbar \Omega_{-}=0.02 \mu \mathrm{eV}$. The ratio $\frac{\Omega_{+}}{\Omega_{-}}$represents the degree to which the polarization is controlled in the experiment. For $\sigma^{-}$polarization, $\hbar \Omega_{+}=0.02 \mu \mathrm{eV}, \hbar \Omega_{-}=0.38 \mu \mathrm{eV}$. The error bars correspond to s.d. for $n=10 . b$, The spin preparation fidelity calculated using the fits in a for each hole spin state. The label identifies the spin shelving state. The error bars correspond to the range in fidelity obtained by varying $T_{1}^{\text {hole }}$ from 0.2 to $1.0 \mathrm{~ms}$. c, Contrast versus applied bias at $1.5 \mathrm{~T}$ for $\sigma^{-}$polarization. The solid red line is the result of the four-level calculation, including both cotunnelling-related (bias-dependent) and phonon-related (bias-independent) hole spin relaxation, while the dashed red line includes only cotunnelling-related hole spin relaxation. The error bars correspond to s.d. for $n=6$. in Fig. 3d. Incoherent processes, spontaneous emission, electron spin relaxation and hole spin relaxation, are included with a master equation for the density matrix. The density matrix is linked to the experiment by computing the quantum dot polarization: the differential transmission signal arises through a destructive interference of the laser and Rayleigh scattered fields. The model is explained in detail in the Supplementary Information. All the parameters for the model are known, apart from $T_{1}^{\text {hole }}$, which we determine by fitting the calculated signal strength to the experimental data (Fig. 4a).

The theory fit to the two data sets in Fig. 4a is excellent over the full two decades of contrast and yields $T_{1}^{\text {hole }}=1 \mathrm{~ms}$ for $B_{\text {nuclei }}^{x y}=21 \mathrm{mT}$. Values for $B_{\text {nuclei }}^{x y}$ for similar dots vary in the literature from 12 to $26 \mathrm{mT}$ (refs 7, and 10-12). Within this range of $B_{\text {nuclei }}^{x y}$ we generate equally good fits to the data with $0.2<T_{1}^{\text {hole }}<1 \mathrm{~ms}$, defining our uncertainty in $T_{1}^{\text {hole }}$. The $T_{1}^{\text {hole }}$ we determine is very similar to that determined from ensemble polarization decay measurements on similar quantum dots at high fields, $B_{\mathrm{ext}}^{z}>1.5 \mathrm{~T}$ (ref. 23), where any hyperfine interaction is suppressed by the mismatch in hole spin and nuclear spin Zeeman energies. In our case, our experiment is most sensitive to $T_{1}^{\text {hole }}$ at low fields where the external magnetic field does not suppress the hyperfine interaction.

The theory perfectly reproduces the repumping experiment in Fig. 3d, in particular the asymmetry about the peak. The electron spin hyperfine interaction couples the two exciton states $|\Uparrow \downarrow, \uparrow\rangle$ and $|\Uparrow \downarrow, \downarrow\rangle$, which are diagonalized with a quantization axis tilted from $\mathbf{z}$. In the tilted basis, the exciton states become $|\Uparrow \downarrow, \tilde{\uparrow}\rangle$ and $|\Uparrow \downarrow, \tilde{\downarrow}\rangle$, and there are two repumping transitions: at $B_{\text {ext }}^{z}=0.25 \mathrm{~T},|\Uparrow\rangle \leftrightarrow|\Uparrow \downarrow, \tilde{\uparrow}\rangle$ is strong and $|\Uparrow\rangle \leftrightarrow|\Uparrow \Downarrow, \tilde{\downarrow}\rangle$ is weak. The asymmetry in the repumping spectrum arises because at large laser energies there is a small contribution from the weak repumping transition. The asymmetry therefore provides further evidence for the coupling of the two exciton states.

We define the fidelity of the hole spin initialization as $\left(\frac{\rho_{22}-\rho_{11}}{\rho_{11}+\rho_{22}}\right)$ for excitation with $\sigma^{+}$polarization and as $\left(\frac{\rho_{11}-\rho_{22}}{\rho_{11}+\rho_{22}}\right)$ for $\sigma^{-}$polarization where $\rho_{11}$ (or $\rho_{22}$ ) is the population of state $|\Downarrow\rangle$ (or $\left.|\Uparrow\rangle\right)$. The experiment is sensitive not to the populations but to the off-diagonal components of the density matrix. We use the theory to link the two. Under the experimental conditions, a slightly elliptically polarized excitation $\left(\sigma^{+}=99.7 \%\right.$; $\sigma^{-}=0.3 \%$ in power $)$, we deduce a fidelity of $99.0 \pm 0.5 \%$ at $B_{\text {ext }}^{z}=0$. The uncertainty arises through the uncertainty in $B_{\text {nuclei }}^{x y}$. The main experimental limitation is at present the polarization: for pure $\sigma^{+}$polarization, the theory predicts an increase in fidelity to above $99.9 \%$. Figure 4 b shows how the fidelity falls as the spin pumping mechanism is gradually turned off with an external magnetic field. We note that these results are not restricted to this particular dot: we have demonstrated high fidelity hole spin preparation on about ten different quantum dots.

We turn to the limiting hole spin relaxation mechanism. Cotunnelling is one possibility. A single hole is trapped in the dot over an applied bias range. At the edges of this voltage plateau, the quantum dot and Fermi energies are nearly degenerate, enabling fast hole spin relaxation through cotunnelling ${ }^{6,7,27}$. Consistent with this, the transmission contrast increases at the plateau edges (Fig. 4b), because cotunnelling now dominates the hole spin relaxation. We can fit the data to determine a cotunnelling spin relaxation time at the plateau edge of $\sim 3 \mu \mathrm{s}$. We then predict the cotunnelling spin relaxation time at the plateau centre to be $\sim 100 \mathrm{~ms}$. This time of $100 \mathrm{~ms}$ is much longer than the $T_{1}^{\text {hole }}$ deduced from the data in Fig. $4 \mathrm{a}$, and demonstrates that cotunnelling is not the fastest hole spin relaxation mechanism in the plateau centre. Instead, it is likely that a combination of spin-orbit and hole-phonon interactions ultimately limits the coherence of the hole $\operatorname{spin}^{22,23}$.

The high-fidelity hole spin initialization demonstrated here opens the way to the generation of arbitrary hole spin states either by electric field induced spin resonance ${ }^{29}$ or by an all-optical process, stimulated 
Raman adiabatic passage ${ }^{30}$. It is tantalizing to note that in the limit in which hole spin relaxation is limited by an interaction with the phonon bath, $T_{2}^{\text {hole }}$ has been predicted ${ }^{4}$ to reach the maximum value of $2 T_{1}^{\text {hole }}$, which would correspond to a millisecond timeframe. In the context of quantum information processing, this would allow many quantum operations to be executed before the hole spin dephases.

\section{Received 5 July; accepted 6 November 2007.}

1. Loss, D. \& DiVincenzo, D. P. Quantum computation with quantum dots. Phys. Rev. A 57, 120-126 (1998).

2. Kroutvar, M. et al. Optically programmable electron spin memory using semiconductor quantum dots. Nature 432, 81-84 (2004).

3. Elzerman, J. M. et al. Single-shot read-out of an individual electron spin in a quantum dot. Nature 430, 431-435 (2004).

4. Golovach, V. N., Khaetskii, A. \& Loss, D. Phonon-induced decay of the electron spin in quantum dots. Phys. Rev. Lett. 93, 016601 (2004)

5. Amasha, S. et al. Measurements of the spin relaxation rate at low magnetic fields in a quantum dot. Preprint at 〈http://arxiv.org/abs/cond-mat/0607110〉 (2006).

6. Atatüre, M. et al. Quantum-dot spin-state preparation with near-unity fidelity. Science 312, 551-553 (2006)

7. Dreiser, J. et al. Optical investigations of quantum-dot spin dynamics. Preprint at 〈http://arxiv.org/abs/0705.3557〉 (2007).

8. Merkulov, I. A., Efros, A. I. L. \& Rosen, M. Electron spin relaxation by nuclei in semiconductor quantum dots. Phys. Rev. B 65, 205309 (2002)

9. Khaetskii, A. V., Loss, D. \& Glazman, L. Electron spin decoherence in quantum dots due to interaction with nuclei. Phys. Rev. Lett. 88, 186802 (2002).

10. Johnson, A. C. et al. Triplet-singlet spin relaxation via nuclei in a double quantum dot. Nature 435, 925-928 (2005)

11. Bracker, A. S. et al. Optical pumping of the electronic and nuclear spin of single charge-tunable quantum dots. Phys. Rev. Lett. 94, 047402 (2005).

12. Braun, P. F. et al. Direct observation of the electron spin relaxation induced by nuclei in quantum dots. Phys. Rev. Lett. 94, 116601 (2005).

13. Koppens, F. H. L. et al. Control and detection of singlet-triplet mixing in a random nuclear field. Science 309, 1346-1350 (2005).

14. Petta, J. R. et al. Coherent manipulation of coupled electron spins in semiconductor quantum dots. Science 309, 2180-2184 (2005).

15. Greilich, A. et al. Mode locking of electron spin coherences in singly charged quantum dots. Science 313, 341-345 (2006)
16. Burkard, G., Loss, D. \& DiVincenzo, D. P. Coupled quantum dots as quantum gates. Phys. Rev. B 59, 2070-2078 (1999).

17. Imamoglu, A., Knill, E., Tian, L. \& Zoller, P. Optical pumping of quantum-dot nuclear spins. Phys. Rev. Lett. 91, 017402 (2003).

18. Coish, W. A. \& Loss, D. Hyperfine interaction in a quantum dot: non-markovian electron spin dynamics. Phys. Rev. B 70, 195340 (2004).

19. Lai, C. W., Maletinsky, P., Badolato, A. \& Imamoglu, A. Knight-field-enabled nuclear spin polarization in single quantum dots. Phys. Rev. Lett. 96, 167403 (2006).

20. Postma, H. W. C., Teepan, T., Yao, Z., Grifoni, M. \& Dekker, C. Carbon nanotube single-electron transistors at room temperature. Science 293, 76-79 (2001).

21. Trauzettel, B., Bulaev, D. V., Loss, D. \& Burkard, G. Spin qubits in graphene quantum dots. Nature Phys. 3, 192-196 (2007).

22. Bulaev, D. V. \& Loss, D. Spin relaxation and decoherence of holes in quantum dots. Phys. Rev. Lett. 95, 076805 (2005).

23. Heiss, D. et al. Observation of extremely slow hole spin relaxation in selfassembled quantum dots. Preprint at $\langle$ http://arxiv.org/abs/0705.1466 $\rangle$ (2007).

24. DiVincenzo, D. P. The physical implementation of quantum computation. Fortschr. Phys. 48, 771-783 (2000).

25. Urbaszek, B. et al. Fine structure of highly charged excitons in semiconductor quantum dots. Phys. Rev. Lett. 90, 247403 (2005).

26. Högele, A. et al. Voltage controlled optics of a quantum dot. Phys. Rev. Lett. 93, 217401 (2004)

27. Smith, J. M. et al. Voltage-control of the spin flip rate of an exciton in a semiconductor quantum dot. Phys. Rev. Lett. 94, 197402 (2005).

28. Gerardot, B. D. et al. Contrast in transmission spectroscopy of a single quantum dot. Appl. Phys. Lett. 90, 221106 (2007)

29. Bulaev, D. V. \& Loss, D. Electric dipole spin resonance for heavy holes in quantum dots. Phys. Rev. Lett. 98, 097202 (2007).

30. Bergmann, K., Theuer, H. \& Shore, B. W. Coherent population transfer among quantum states of atoms and molecules. Rev. Mod. Phys. 70, 1003-1025 (1998).

Supplementary Information is linked to the online version of the paper at www.nature.com/nature.

Acknowledgements This work was funded by the EPSRC (UK), the DFG and the EU (SANDiE). B.D.G. thanks the Royal Society of Edinburgh for financial support.

Author Information Reprints and permissions information is available at www.nature.com/reprints. Correspondence and requests for materials should be addressed to B.D.G. (b.d.gerardot@hw.ac.uk) or R.J.W. (r.j.warburton@hw.ac.uk) 